11

\title{
Измерение расстояния между излучателем и приемником в беспроводном канале связи при помощи сверхширокополосных хаотических радиоимпульсов
}

\author{
(C) Е.В. Ефремова ${ }^{1,2}$, А.С. Дмитриев ${ }^{1,2}$, Л.В. Кузьмин ${ }^{1,2}$ \\ ${ }^{1}$ Институт радиотехники и электроники им. В.А. Котельникова РАН, Москва, Россия \\ ${ }^{2}$ Московский физико-технический институт (Национальный исследовательский университет), Долгопрудный, \\ Московская обл., Россия \\ E-mail: efremova@cplire.ru
}

Поступило в Редакцию 6 мая 2019 г.

В окончательной редакции 6 мая 2019 г.

Принято к публикации 13 мая 2019 г.

Рассматривается возможность беспроводного измерения расстояния при помощи сверхширокополосных хаотических радиоимпульсов на основе статистического анализа сигнала. Приводятся результаты экспериментальной апробации предложенного подхода.

Ключевые слова: измерение расстояния, сверхширокополосные хаотические радиоимпульсы, беспроводной канал связи.

DOI: 10.21883/PJTF.2019.17.48214.17865

В условиях активного развития новых коммуникационных направлений, таких как Интернет вещей, межмашинное взаимодействие (М2М), робототехника, особую актуальность приобрела задача беспроводного локального позиционирования в закрытых помещениях без использования систем глобального позиционирования общего назначения.

За последние годы большую популярность получили измерение расстояний и позиционирование объектов при помощи сигналов различных типов. Системы позиционирования реализованы на таких технологиях, как WiFi, Bluetooth, BLE, ZigBee, UWB, акустические технологии и др. Основные способы измерения расстояния в беспроводных системах следующие: по уровню принимаемого сигнала (RSSI), по времени его распространения (TOA, TDOA, RTOF и пр.), по его фазе и др. Подробный обзор можно найти в работах $[1,2]$. В зависимости от используемой технологии, метода измерения и дополнительных средств обработки сигналов точность определения расстояния колеблется от $15 \mathrm{~cm}$ до нескольких метров. Наибольшую точность демонстрируют системы, основанные на сверхширокополосных (СШП) ультракоротких импульсах, использующие для измерения расстояния время распространения сигнала, но они же имеют и наиболее сложную аппаратную реализацию.

Альтернативным типом СШП-сигнала являются хаотические СШП-радиоимпульсы, в которых сверхширокая полоса достигается не за счет укорочения излучаемого импульса, а за счет формирования шумоподобного сигнала, который в силу способа генерации уже занимает сверхширокую полосу частот $[3,4]$. Типичная длительность хаотических СШП-радиоимпульсов намного больше, чем длительность ультракоротких импульсов ( 100 ns для хаотических радиоимпульсов против
0.1-1 ns для ультракоротких), поэтому их затруднительно использовать как средство определения расстояния, основанное на измерении времени распространения, так как точность фиксации прихода хаотических радиоимпульсов приемником составляет долю его длительности, что при точности фиксации в $10 \mathrm{~ns}$ даст ошибку измерения $\sim 3 \mathrm{~m}$. Это уступает по точности возможностям, которые предоставляет использование ультракоротких импульсов.

Вместе с тем известно [5], что хаотические СШПрадиоимпульсы практически не подвержены многолучевому замиранию в беспроводном канале, что можно использовать как физическую основу для измерения расстояния посредством измерения мощности таких импульсов в приемнике, опираясь на закон затухания мощности сигнала с расстоянием. Как показали эксперименты [6], СШП-сигналы обеспечивают гладкую зависимость мощности принимаемого сигнала от расстояния по степенному закону $1 / d^{n}$, в то время как, например, для узкополосных сигналов, которые подвержены сильному многолучевому замиранию (используются, в частности, в технологии ZigBee), это не так [7]. В узкополосных системах многолучевое распространение приводит к большим вариациям измеряемой приемником мощности узкополосного сигнала, что дает рост ошибки в измерении расстояния.

С практической точки зрения актуальность применения хаотических радиоимпульсов для измерения расстояния также мотивируется тем, что они являются опциональным решением в коммуникационных системах стандарта IEEE 802.15.4а и одним из основных решений стандарта IEEE 802.15.6. В существующей аппаратуpe $[8,9]$ хаотические радиоимпульсы применяются для передачи информации, поэтому практически интерес- 


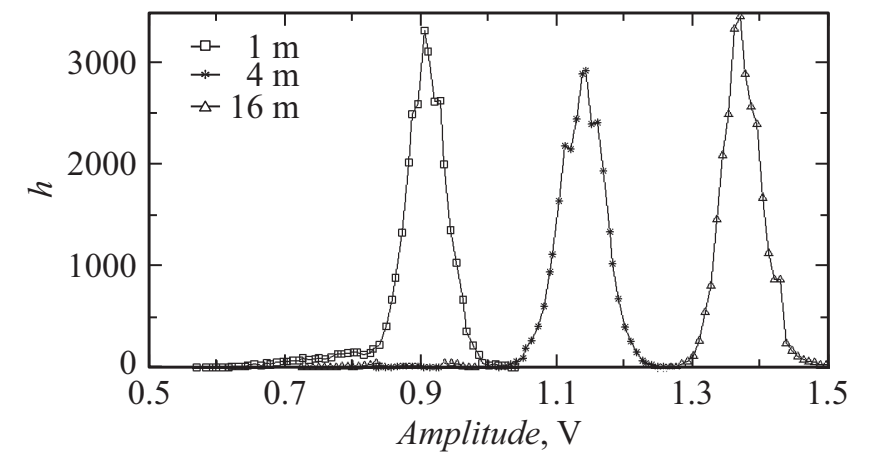

Рис. 1. Распределение амплитуд огибающей хаотических радиоимпульсов в приемнике при различных расстояниях $d$ между излучателем и приемником (указаны на рисунке).

ным является создание способа измерения расстояния, который был бы частью процесса беспроводного обмена данными между устройствами.

В работе исследуется возможность измерения расстояния при помощи хаотических СШП-радиоимпульсов, основанная на измерении относительного уровня мощности сигнала, приходящего в приемник, при помощи статистической оценки его характеристик.

Идея метода состоит в том, что передающее устройство (излучатель) формирует и передает в эфир поток хаотических радиоимпульсов. Приемное устройство детектирует поступающие радиоимпульсы, определяет уровень их мощности по их огибающей и вычисляет число принятых радиоимпульсов, мощность которых больше некоторого заданного значения. Опираясь на это и на закон затухания мощности сигнала в канале, можно вычислить расстояние, которое они прошли от излучателя до попадания в приемник.

Пусть $P_{d} \sim 1 / d^{n}$ - затухание сигнала на расстоянии $d$ между приемником и излучателем, а $n-$ показатель затухания в реальном беспроводном канале. Тогда $[10] P_{d}$ может быть рассчитано как

$$
P_{d}=P_{0}+10 n \lg \left(d / d_{0}\right),
$$

где $P_{0}$ - затухание сигнала на расстоянии $d_{0}$.

При экспериментальной реализации для наблюдения доступны три величины: $P_{d}, P_{0}$ и $d_{0}$. Величины $P_{d}$ и $P_{0}$ можно измерять при помощи логарифмического детектора, который формирует на своем выходе сигнал с амплитудой $A_{d}$, пропорциональной логарифму мощности на входе приемника, т. е. пропорциональной $P_{d}$. Величина $d_{0}$ в эксперименте определяется одновременно с $P_{0}$.

Затухание $P_{\mathrm{ADD}}=10 n \lg \left(d / d_{0}\right)$ определяется как разность $10 n \lg \left(d / d_{0}\right)=P_{d}-P_{0}$ путем сравнения амплитуды $A_{d}$ импульсов с мощностью $P_{d}$ на входе логарифмического детектора с амплитудой $A_{0}$ сигнала, соответствующего мощности $P_{0}$ на входе приемника на расстоянии $d_{0}$ от излучателя. Зная зависимость напряжения на выходе детектора от мощности входного сигнала [11], получаем

$$
P_{d}-P_{0}=\left(A_{d}-A_{0}\right) / h
$$

где $h$ - крутизна характеристики детектора.

Тогда расстояние $d$ определяется как

$$
d=d_{0} \cdot 10^{\left(P_{d}-P_{0}\right) /(10 n)}=d_{0} \cdot 10^{\left(A_{d}-A_{0}\right) /(10 n h)} .
$$

В свободном пространстве $n=2$, в реальном беспроводном канале с прямым лучом $n<2$, без прямого луча $n>3[12]$.

Измерение амплитуд $A_{d}$ огибающей радиоимпульсов в приемнике реализуется посредством сравнения этих амплитуд с некоторым пороговым значением $A_{T}$ и подсчета числа импульсов, обнаруживаемых путем такого сравнения, для нахождения границы, при которой приемник перестает фиксировать импульсы.

Мощность хаотических СШП-радиоимпульсов варьируется от импульса к импульсу. Распределение амплитуд их огибающих в приемнике, измеренное в эксперименте при различных расстояниях между излучателем и приемником, показано на рис. 1.

Ширина распределения амплитуд импульсов на различных расстояниях от излучателя остается приблизительно одинаковой. Поскольку для вычисления расстояния используется разница амплитуд $\left(A_{d}-A_{0}\right)$, это дает возможность зафиксировать уровень ошибки обнаружения радиоимпульсов для реперного расстояния $d_{0}$ и расстояния $d$ в точке приема, оценить пороговое значение $A_{T}$ для этих расстояний при заданном уровне ошибки и на основании этого получить оценку расстояния.

Для подтверждения изложенных идей был реализован эксперимент по схеме, показанной на рис. 2, с использованием сверхширокополосных прямохаотических приемопередатчиков (СШП ПП) [9], которые играли роль излучателя и приемника хаотических радиоимпульсов. Диапазон рабочих частот СШП ПП находится в пределах 3-5 GHz. В ходе эксперимента устройства располагались в условиях прямой видимости. Положение устройства-приемника было фиксировано, положение передатчика менялось с шагом 0.5-1 m вдоль прямой.

В эксперименте устройство $T_{X}$ формировало поток хаотических радиоимпульсов в виде пачек (пакетов), снабженных служебной информацией (количество импульсов, контрольная сумма), необходимой для определения правильности приема пакета. В процессе приема в устройстве $R_{X}$ поступающий сигнал усиливается, пропускается через логарифмический детектор с характеристикой [11] и сравнивается с пороговым значением $A_{T}$ по следующему правилу: если амплитуда огибающей выше этого значения, то импульс считается принятым; если ниже, то нет. Далее подсчитывается число импульсов в пакете, которые удовлетворяют этому условию. Данный процесс эквивалентен схеме приема пакетов в ходе обмена информацией между приемопередатчиками [8]. 

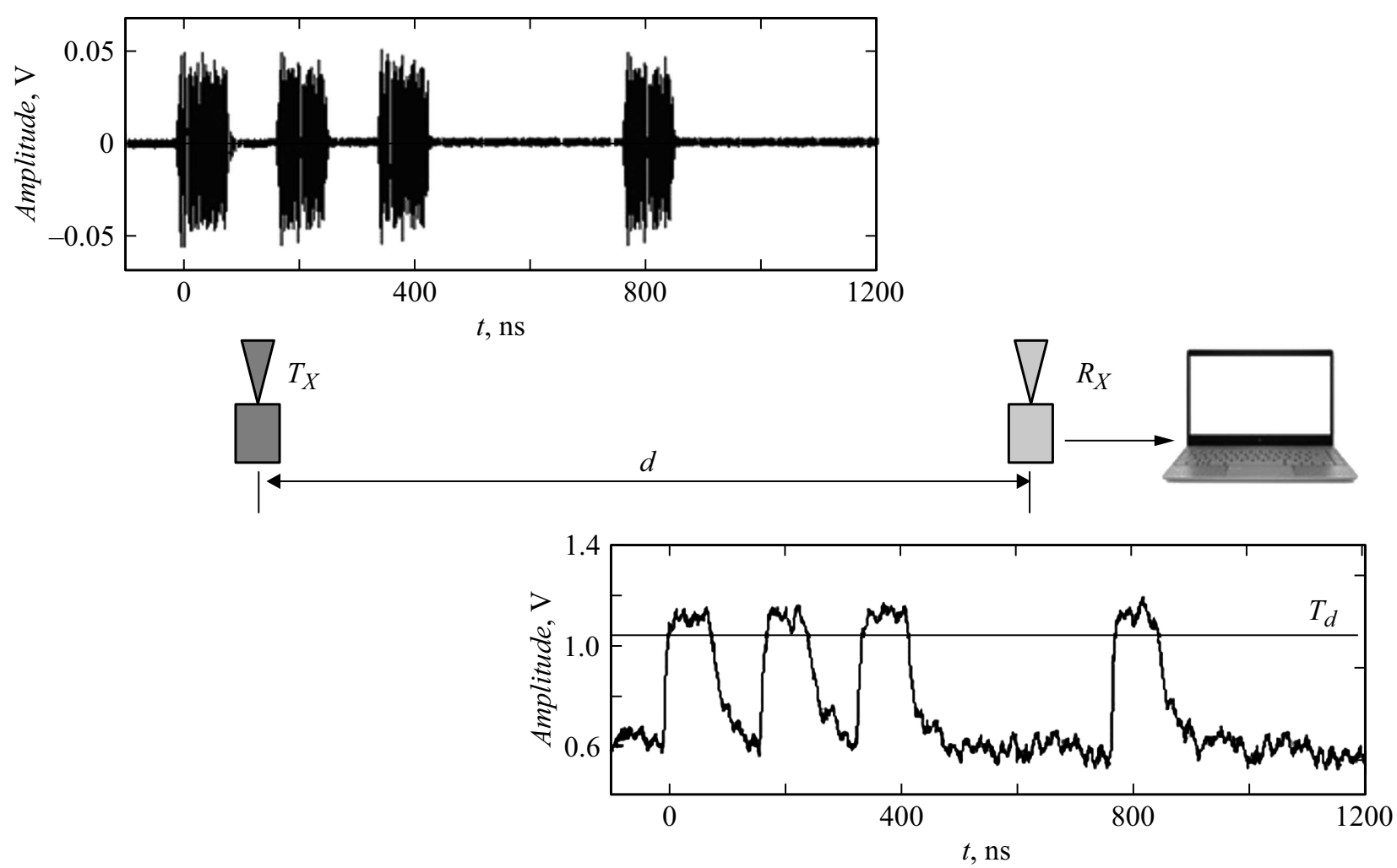

Рис. 2. Схема эксперимента.
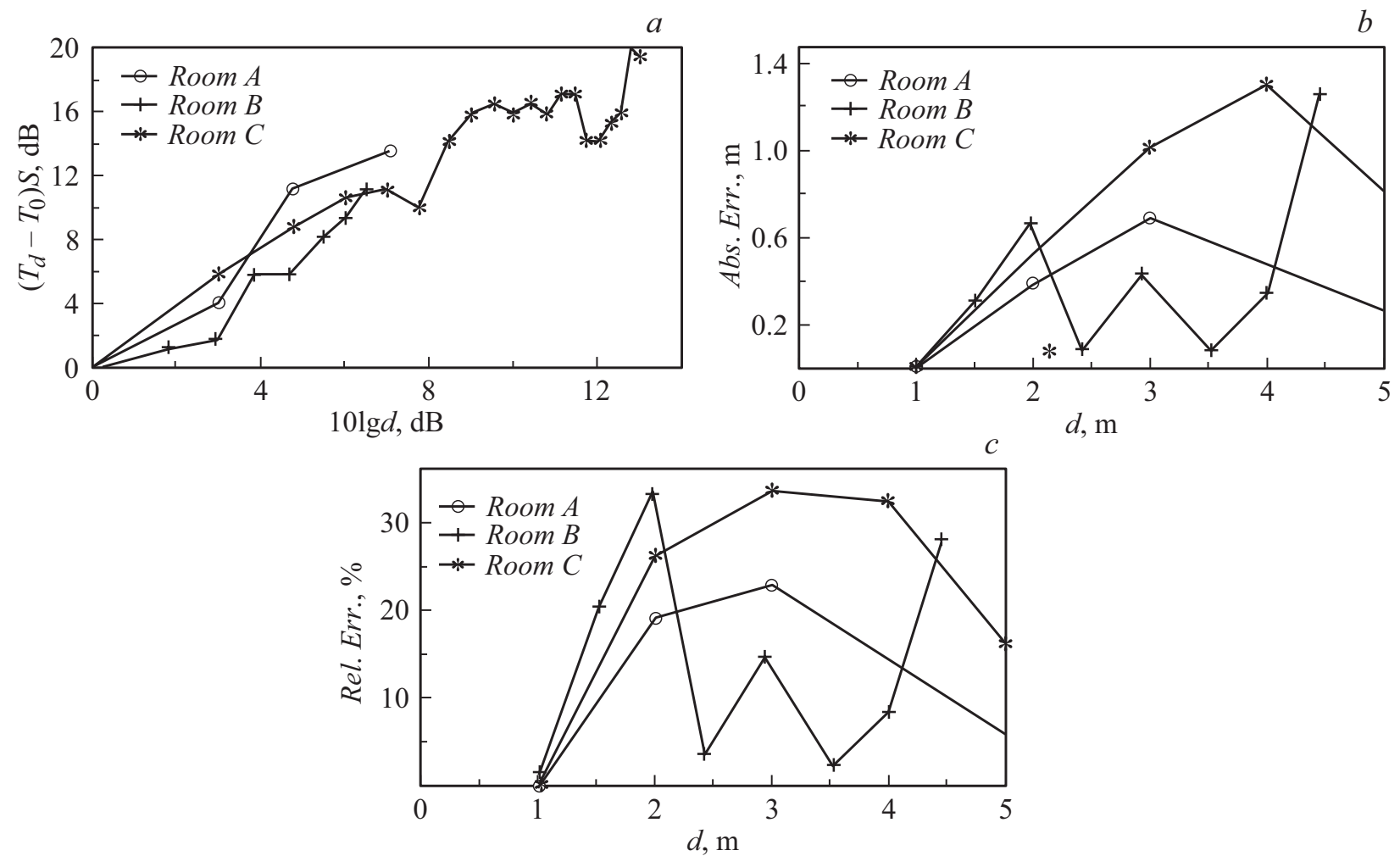

Рис. 3. $a-$ семейство зависимостей $\left(T_{d}-T_{0}\right) S$ от $10 \lg d$ в экспериментах; $b-$ семейство зависимостей абсолютных ошибок (Abs. Err.) от расстояния для различных экспериментов; c- семейство зависимостей относительных ошибок (Rel. Err.) от расстояния для различных экспериментов. 
Результаты определения коэффициента наклона прямой $n$

\begin{tabular}{c|c|c}
\hline $\begin{array}{c}\text { PER }=99 \% \\
(\text { помещение } A)\end{array}$ & $\begin{array}{c}\text { PER }=90 \% \\
(\text { помещение } B \text { ) }\end{array}$ & $\begin{array}{c}\text { PER }=99 \% \\
\text { (помещение } C \text { ) }\end{array}$ \\
\hline $1.97 \pm 0.11$ & $1.48 \pm 0.08$ & $1.46 \pm 0.02$
\end{tabular}

Далее подсчитывалась доля пакетов $P_{B}(d)$, принятых с ошибкой (PER - отношение числа ошибочно принятых пакетов к общему числу пакетов), для заданного расстояния $d$ между излучателем и приемником.

Для каждого расстояния $d$ уровень порогового напряжения $A_{d}$ выбирался так, чтобы достигался желаемый уровень $P_{B}(d)$ пакетных ошибок. Итогом эксперимента стала зависимость пороговых напряжений $A_{d}$ от расстояния $d$ при заданном уровне $P_{B}(d)$.

Эксперимент был проведен в трех помещениях ИРЭ им. В.А. Котельникова РАН: помещения $A$ и $B-$ рабочие кабинеты, помещение $C-$ коридор. Для помещения $B$ была получена зависимость порога от расстояния для PER, равного $90 \%$, для помещений $A$ и $C$ PER $=99 \%$.

В эксперименте значения порога могли принимать одно из 256 значений $T_{d}$ (задаваемых цифро-аналоговым преобразователем), соответствующих изменению $A_{d}$ в пределах $[0,3.3] \mathrm{V}$. Точность задания порогового напряжения составляла $k=3.3 / 255=12.9 \mathrm{mV}$. Крутизна характеристики детектора $h=-22 \mathrm{mV} / \mathrm{dB}$ [11]. Одному шагу порога соответствует изменение мощности на $S=k / h \approx-0.59 \mathrm{~dB}$.

Из формулы (3) следует, что преобразование порогового значения $T_{d}$ в расстояние между излучателем и приемником осуществляется как

$$
d=d_{0} \cdot 10^{\left(T_{d}-T_{0}\right) k /(10 n h)},
$$

где $T_{0}, T_{d}$ - значения порога в реперной точке (на расстоянии $d_{0}$ ) и на расстоянии $d$ (при заданном значении уровня ошибки) соответственно, $k-$ шаг изменения амплитуды (в вольтах) при изменении значения порога на единицу $\left(A_{T}=T_{d} k\right)$.

С учетом теоретической зависимости (4), в которой $d_{0}=1 \mathrm{~m}$, после логарифмирования имеем

$$
\left(T_{d}-T_{0}\right) S=10 n \lg d .
$$

Если по оси абсцисс отложить измеренные значения $10 \lg d$, а по оси ординат - значения $\left(T_{d}-T_{0}\right) S$ (где $S=-0.59 \mathrm{~dB}$ ), то зависимость $10 \lg d$ от $\left(T_{d}-T_{0}\right) S$ должна быть линейной с коэффициентом наклона прямой $n$. Построенные зависимости приведены на рис. $3, a$.

Для определения коэффициента наклона прямой осуществлялась аппроксимация экспериментальных значений методом наименьших квадратов в координатах $\left(10 \lg d,\left(T_{d}-T_{0}\right) S\right)$ для всех экспериментальных серий на рис. 3, $a$. Результаты определения $n$ приведены в таблице.
Для $\mathrm{PER}=99 \%$ в помещении $C$ для расстояний от 0 до $5 \mathrm{~m}$ зависимость хорошо аппроксимируется прямой. Отклонение от линейной зависимости при бо́льших расстояниях связано с взаимодействием между прямым лучом и переотраженными сигналами. Подробнее этот эффект рассмотрен в [6].

На основе вычисленных значений $n$ были получены оценки для расстояний между излучателем и приемником и зависимости абсолютной (рис. $3, b$ ) и относительной (рис. $3, c$ ) ошибок измерений от расстояния.

Средняя относительная ошибка для разных помещений на расстоянии до $5 \mathrm{~m}$ составила $15-18 \%$ (помещения $B, A$ ). Для помещения $C$ на расстоянии до $20 \mathrm{~m}$ ошибка возрастает до $29 \%$.

Таким образом, в работе предложен и экспериментально апробирован метод измерения расстояния между излучателем и приемником в беспроводном канале связи при помощи сверхширокополосных хаотических радиоимпульсов, основанный на измерении статистических характеристик принятого сигнала. По полученным результатам метод сопоставим с современными технологиями определения местоположения, при этом технически является существенно более простым и не требует дополнительных возможностей от существующей аппаратуры помимо операций, связанных с приемом и обработкой СШП-сигнала в ходе беспроводной передачи информации.

\section{Финансирование работы}

Работа выполнена в рамках государственного задания Института радиотехники и электроники им. В.А. Котельникова РАН.

\section{Конфликт интересов}

Авторы заявляют, что у них нет конфликта интересов.

\section{Список литературы}

[1] Zafari F., Gkelias A., Leung K.K. // arXiv:1709.01015v3. 16 Jan 2019.

[2] Alarifi A., Al-Salman A., Alsaleh M., Alnafessah A., Al-Hadhrami S., Al-Ammar M.A., Al-Khalifa H.S. // Sensors. 2016. V. 16. N 5. P. 707.

[3] Дмитриев А.С., Ефремова Е.В., Панас А.И., Максимов Н.А. Генерация хаоса. М.: Техносфера, 2012. 424 с.

[4] Дмитриев А.С., Ефремова Е.В. // Письма в ЖТФ. 2016. T. 42. B. 24. C. $49-57$.

[5] Андреев Ю.В., Дмитриев А.С., Лазарев В.А. СШП прямохаотическая передающая панель. Теория и эксперимент // Тр. науч. конф. „V Всероссийские Армандовские чтения“". Муром, 2015. С. 211-215.

[6] Гуляев Ю.В., Дмитриев А.С., Лазарев В.А., Мохсени Т.И., Попов М.Г. // Радиотехника и электроника. 2016. Т. 61. № 8. C. 1-8. 
[7] Поникар А.В., Евсеев О.В., Анщиперов В.Е., Мансуров Г.К. Исследование возможности локального позиционирования в беспроводных сетях IEEE 802.15.4 // IV Bcерос. конф. „Радиолокация и радиосвязь“. М.: ИРЭ РАН, 2010. C. $914-918$.

[8] Дмитриев А.С., Ефремова Е.В., Клецов А.В., Кузьмин Л.В., Лактюшкин А.М., Юркин В.Ю. // Радиотехника и электроника. 2008. Т. 53. № 10. С. 1278-1289.

[9] Дмитриев А.С., Герасимов М.Ю., Ииков В.В., Лазарев В.А., Попов М.Г., Рыжсов А.И. // Радиотехника и электроника. 2017. Т. 62. № 4. С. 1-10.

[10] Скляр Б. Цифровая связь: теоретические основы и практическое применение. Пер. с англ. 2-е изд. М.: Изд. дом „Вильямс“, 2004. 1104 с.

[11] Analog Device Data Sheet for „AD8317 $1 \mathrm{MHz}-10 \mathrm{GHz}$ 50 dB High Precision Logarithmic Detector“; http://www.analog.com

[12] Molisch A.F. // Proc. of the IEEE. 2009. V. 97. N 2. P. 353-371. 